\title{
Single Mode Theory for Impedance Eduction in Large-Scale Ducts with Grazing Flow
}

\author{
Willie R. Watson*, Carl H. Gerhold, and Michael G. Jones ${ }^{\ddagger}$ \\ NASA Langley Research Center, Hampton, VA 23681 \\ Jason C. June $e^{\S}$ \\ University of Florida, Gainesville, FL 32605
}

\begin{abstract}
An impedance eduction theory for a rigid wall duct containing an acoustic liner with an unknown impedance and uniform grazing flow is presented. The unique features of the theory are: 1) non-planar waves propagate in the hard wall sections of the duct, 2) input data consist solely of complex acoustic pressures acquired on a wall adjacent to the liner, and 3) multiple higher-order modes may exist in the direction perpendicular to the liner and the opposite rigid wall. The approach is to first measure the axial propagation constant of a dominant higher-order mode in the liner sample section. This axial propagation constant is then used in conjunction with a closed-form solution to a reduced form of the convected Helmholtz equation and the wall impedance boundary condition to educe the liner impedance. The theory is validated on a conventional liner whose impedance spectrum is educed in two flow ducts with different cross sections. For the frequencies and Mach numbers of interest, no higher-order modes propagate in the hard wall sections of the smaller duct. A benchmark method is used to educe the impedance spectrum in this duct. A dominant higher-order vertical mode propagates in the larger duct for similar test conditions, and the current theory is applied to educe the impedance spectrum. Results show that when the theory is applied to data acquired in the larger duct with a dominant higher-order vertical mode, the same impedance spectra is educed as that obtained in the small duct where only the plane wave mode is present and the benchmark method is used. This result holds for each higher-order vertical mode that is considered.
\end{abstract}

\section{Nomenclature}

$A_{m n}, K_{m n} \quad=$ complex mode coefficients, complex axial propagation constants

$c_{0}, \rho_{0} \quad=$ ambient sound speed, ambient density of air

$f, \omega=$ excitation frequency, angular frequency $(2 \pi f)$

$H, L, W, d \quad=$ height of flow duct, length of flow duct, width of flow duct, depth of liner cavity

$i, k=$ unit imaginary number $(\sqrt{-1})$, free space wavenumber $\left(\frac{\omega}{c_{0}}\right)$

$L_{1}, L_{2} \quad=$ axial location of the leading edge of liner, axial location of the trailing edge of liner

$M, \zeta=$ uniform mean flow Mach number, normalized liner impedance

nmax,$\quad=$ number of normal modes in the horizontal direction, dimensional time

$p, P \quad=3-\mathrm{D}$ acoustic pressure field, normal acoustic pressure mode

$x, y, z \quad=$ vertical coordinate, horizontal coordinate, axial coordinate

$\mathfrak{R}, \mathfrak{I}=$ real part of a complex quantity, imaginary part of a complex quantity

subscripts:

$m, n=$ dominant vertical mode order, horizontal mode order

\footnotetext{
*Senior Research Scientist, Computational AeroSciences Branch, Mail Stop 128, Willie.R.Watson@ NASA.Gov, Associate Fellow AIAA.

${ }^{\dagger}$ Senior Research Scientist, Aerocoustics Branch, Mail Stop 461, Carl.H.Gerhold@NASA.Gov, Associate Fellow AIAA.

${ }^{\ddagger}$ Senior Research Scientist, Structural Acoustics Branch, Mail Stop 463, Michael.G.Jones@NASA.Gov, Associate Fellow AIAA.

${ }^{\S}$ LARSS summer Intern and PhD candidate.
} 


\section{Introduction}

NCREASINGLY stringent international noise constraints have resulted in continued emphasis on development of improved technologies to reduce the overall level of fan noise radiated to communities that surround airports. Although local-reacting sound absorbing structures (referred to as acoustic liners) mounted in the aircraft engine nacelles currently provide significant fan-noise reduction, further optimization is required to increase their noise reduction capacity and bandwidth. The critical intrinsic parameter used in this optimization is the local-reacting acoustic impedance of the liner. ${ }^{1}$ Therefore, an accurate knowledge of this quantity is critical for the design of quieter aircraft. The local-reacting impedance of a nacelle liner is defined as the ratio of the acoustic pressure to the normal component of acoustic particle velocity at the surface of the liner. A direct, simultaneous measurement of both of these quantities at the liner surface is problematic at best. Instead, a number of indirect methods have been developed to determine the local-reacting acoustic impedance of the liner.

For over 30 years, the NASA Langley Research Center has invested a significant effort in the development of indirect impedance prediction tools and experimental rigs for the evaluation of acoustic liner impedance. ${ }^{2-14}$ This has included the development of prediction tools to account for the effects of both uniform flow and shear flow profiles in the duct. These impedance eduction methods may be further divided into non-modal methods that are based on the conventional finite element method ${ }^{2,3,5,6,14}$ and the single mode methods (SMM) ${ }^{4,7-10,12-14}$ that are based on single mode analysis. All of these methods have the restriction that they are applicable to a rectangular waveguide with rigid walls, with the test sample inserted into one of these walls. They each have the additional restriction that the sound field in the coordinate direction perpendicular to the two opposite rigid walls is uniform.

The simplest of the impedance eduction methods is the SMM. This method involves measuring the axial wavenumber for a single dominant mode in the rectangular waveguide. The measured axial wavenumber for the mode is used, in conjunction with the solution to the convected (or sheared flow) wave equation to educe the impedance of the liner., ${ }^{4-10,12-14}$ The SMM is applicable in a very straightforward manner to a situation for which a single unidirectional mode propagates over the liner. This method breaks down when end effects (due to impedance discontinuities at the leading and trailing edges of the liner) propagate over the extent of the liner, when non-planar waves propagate in the hard wall sections of waveguide, or when more than one mode is dominant over the extent of the liner. ${ }^{4}$ Recent development has given focus on the SMM a new impetus. For example, the use of the Prony method ${ }^{12-14}$ on a measured acoustic field now means that a single unidirectional mode component can be extracted from a complicated acoustic field. The SMM can then be applied to the single unidirectional mode component of the acoustic field to extract the unknown impedance. Further, more sophisticated measurement devices such as the NASA Langley Curved Duct Test Rig (CDTR) ${ }^{15}$ allows control and analysis of high order modes in the cross-sectional directions.

Up until now the use of the SMM at the NASA Langley Research Center has been for the NASA Langley Grazing Flow Impedance Tube (GFIT) and its predecessor, the Grazing Incidence Tube (GIT). In these measurement apparatuses the sound field in the rigid wall sections consists of only the plane wave mode in both coordinate directions, so the analysis is greatly simplified, and the impedance can be educed without the complication of an iterative process as with the non-modal methods. The goal of this research effort is to develop a corresponding single mode theory for impedance eduction in a large-scale duct, such as the CDTR, where a higher-order mode is dominant in the coordinate direction perpendicular to the two opposite rigid walls. In this paper, a higher-order mode theory is developed and then validated using a conventional liner and test data acquired in the CDTR. First, the impedance spectrum of a conventional liner is educed using test data acquired in the GFIT, for which only the plane wave mode is present (i.e., cuton) in the hard wall sections of the duct. This impedance spectrum is educed using a benchmark method. ${ }^{3}$ A similar liner is then mounted in the CDTR and tested at the same frequencies and similar Mach numbers as in the GFIT. Because of the much larger dimensions of the CDTR, higher-order modes are cut on in the hard wall sections of the CDTR so that the higher-order mode theory presented in this paper is needed to educe the impedance spectrum. Validation of the method is achieved by demonstrating that the same impedance spectrum is educed in both ducts for similar test conditions.

For over 60 years it has been assumed that each normal mode in a flow duct sees the same impedance at the liner surface. This assumption greatly simplifies acoustic analysis and is fundamental to all mode analysis techniques. ${ }^{1}$ However, up to now, detailed measurements necessary to validate this assumption have not been available. A secondary goal of this research is therefore to isolate individual higher-order vertical modes in a flow duct and to demonstrate (using measured data) that the educed impedance (to within a reasonable tolerance) is independent of the choice of higher-order source modes.

This paper is organized as follows. Section II describes the test facility and test setup. Section III describes the CDTR flow duct and coordinate system for which the analysis is performed. The governing equations and boundary conditions are presented in Section IV. Section V summarizes the impedance eduction procedure, and the test liner is 
described in section VI. Results of the validation exercise are presented in Section VII and concluding remarks, along with recommendations for future research are presented in Section VIII.

\section{Description of Test Facility and Test Set-up}

The CDTR is an open loop wind tunnel that uses a fan to draw unconditioned atmospheric air through the test section, as shown in Fig. 1. The flow delivery system consists of an inlet bellmouth in a shed to prevent debris/water ingestion, a $1.52 \mathrm{~m} \times 0.76 \mathrm{~m}$ vertical duct, a $90^{\circ}$ bend with a $1.14 \mathrm{~m}$ average radius, and a $1.52 \mathrm{~m} \times 0.76 \mathrm{~m}$ to $0.76 \mathrm{~m} \times 0.76 \mathrm{~m}$ transition (transition 1). Downstream of this transition are a flow conditioning section with screens and honeycomb, a contraction to a $0.15 \mathrm{~m} \times 0.38 \mathrm{~m}$ cross section, and an acoustic driver section. These are followed by microphone and traverse sections, used to measure acoustic pressures and flow profiles upstream of the liner test section. Downstream of the liner is a second microphone section and a diffuser that feeds into a transition to a $0.99 \mathrm{~m} \times 0.99 \mathrm{~m}$ duct, through a resonator/muffler, and to a fan. The fan is a centrifugal fan rated at $1,416 \mathrm{~m}^{3} / \mathrm{min}$ and driven by a $447 \mathrm{~kW}$ motor. A parallel-baffle muffler and resonator are designed to reduce fan-generated noise to $10 \mathrm{~dB}$ below the expected flow noise in the test section at Mach 0.5. Figure 2 shows a representation of the test section of the CDTR. Flow is from left to right. Figure 3 shows the liner test section with the straight liner installed on the right sidewall and the hard wall opposite. The lid, which contains the linear microphone array, has been removed for this photograph, but is shown in Fig. 4. Figure 2 shows the loudspeaker source section, the upstream microphone section, the liner test section, and the downstream microphone section. Sound in the duct is generated by an array of 16 loudspeakers. The magnitude and phase of the voltage signal to each loudspeaker is controlled such that a selected mode can be generated in the duct. A subset of the upstream microphone array is used as the control to adjust the signals to the loudspeakers. The microphone and loudspeaker arrays and the sound control system are described in previous papers. ${ }^{15,16}$ The recorded signals from the upstream and downstream microphone arrays are analyzed to determine the mode distribution of the sound in the duct incident upon and discharged from the liner test section. The control system is designed not only to generate a selected mode but also to suppress all other modes in the duct. Thus, the sound incident on the liner is predominantly composed of the selected mode, which is at least $10 \mathrm{~dB}$ greater than any other mode in the duct. This was demonstrated previously by one of the authors. ${ }^{17}$

The sound wave incident on the liner test section consists of a tone generated in the duct at $130 \mathrm{~dB}$. The wave is controlled to have either symmetric (horizontal order 0 and 2) or asymmetric (horizontal order 1) mode-shape in combination with up to five vertical mode orders. The modes are designated as (vertical, horizontal) throughout this report. For this test, either of the $(0,0),(1,0)$, and $(2,0)$ modes is generated in the hard wall section upstream of the liner test section. The liner test section is a duct of $0.15 \mathrm{~m} \times 0.38 \mathrm{~m}$ cross section; and the liner sample, which is assumed uniform and local-reacting, comprises a portion of the right sidewall of the duct. The wall opposite the liner is fully rigid, as are the top and bottom walls of the duct. Although the design of the CDTR allows for curvature along the flow path, the configuration for this test contains no curvature. On the top surface of the flow path, adjacent to the liner, is a linear array of microphones that span the length of the liner.

\section{Description of Flow Duct and Coordinate System}

A schematic of the portion of the CDTR that is used in this analysis is shown in Fig. 5. The mean flow field is assumed constant with Mach number $M$, directed from left to right along the axis of the duct. Here, the height, $H$, of the duct is $0.38 \mathrm{~m}$, and the width, $W$, is $0.15 \mathrm{~m}$. The upper, lower, and left sidewalls of the flow duct are acoustically rigid, and the liner sample is located over a portion of the right sidewall. This paper uses a right-handed coordinate system $(x, y, z)$ where $y$ is the vertical, $x$ is the horizontal, and $z$ is the axial coordinate, as shown in the figure. The origin of the coordinate system is in the lower right corner of the flow duct, $0.58 \mathrm{~m}$ upstream of the leading edge of the liner $\left(L_{1}=0.58 \mathrm{~m}\right)$ as shown. This axial location corresponds to the location of the furthest upstream microphone in the rigid wall section upstream of the liner. Further, the exit plane is located $0.58 \mathrm{~m}$ downstream of the trailing edge of the liner $\left(L-L_{2}=0.58 \mathrm{~m}\right)$; and its location coincides with the furthest downstream microphone in the rigid wall section downstream of the liner. The liner sample is $0.81 \mathrm{~m}$ in length $\left(L_{2}-L_{1}=0.81 \mathrm{~m}\right)$, and its unknown normalized impedance is assumed constant and denoted by $\zeta$. Throughout this paper all impedances are normalized with the characteristic impedance, $\rho_{0} c_{0}$, of the air flowing in the duct. As show in Fig. 5, there is a single linear array of microphones mounted in the lid of the CDTR. This array consists of 32 equally spaced microphones located $2 W / 3$ units from the right sidewall (i.e., $x=2 \mathrm{~W} / 3$ ). The location of the axial array of microphones was chosen to avoid a pressure null. The first microphone is located at the leading edge of the liner, and the last microphone is located 25.4 $\mathrm{mm}$ upstream of the trailing edge of the liner. The goal of the analysis is to educe the unknown normalized impedance, 
$\zeta$, of the test sample by using acoustic pressure data obtained from the lid microphones. The unique features of the CDTR are: 1) higher-order modes are cut on in the direction perpendicular to the two rigid walls, 2) the upstream energy in these higher-order modes can be controlled and measured, and 3) acoustic pressure measurements in the liner test section are available only on the wall adjacent to the liner (i.e., the lid). The tools developed for impedance eduction in the GFIT (where only the plane wave mode is cut on in the direction perpendicular to the two rigid walls) are not directly transferable to the CDTR (where higher-order modes are cut on between the two rigid walls). Thus, an extension of these impedance eduction tools is required and is presented in this paper.

\section{Governing Equation and Boundary Conditions}

Under the assumptions made in this paper (i.e., the sound energy residing in a dominant vertical mode, a uniform mean flow field, and a uniform impedance liner), the acoustic pressure field in the liner sample section can be expanded as a series of normal duct modes

$$
p(x, y, z)=\sum_{n=0}^{n m a x} A_{m n} P_{m n}(x) \cos \left(\frac{m \pi y}{H}\right) e^{-i K_{m n} z}
$$

Here $m$ is the dominant vertical mode order, and nmax is chosen large enough so that all cuton modes and a few cutoff modes are included in the mode series. The $A_{m n}$ 's are the complex mode coefficients in the liner test section, each $P_{m n}(x)$ is the normal acoustic pressure mode, and each $K_{m n}$ is the axial propagation constant that can be determined from the microphone data in the lid. Right-moving waves decay as they propagate along the positive $z$ axis (i.e., $\mathfrak{I}\left(K_{m n}\right)<0$ ) whereas left-moving waves decay as they propagate along the negative $z$ axis (i.e., $\mathfrak{I}\left(K_{m n}\right)>0$ ). If $K_{m n}$ is wholly real $\left(\mathfrak{I}\left(K_{m n}\right)=0\right)$, the acoustic wave is propagating without decay. In this situation, the axial propagation constant of the right moving waves will have a positive real part (i.e., $\mathfrak{R}\left(K_{m n}\right)>0$ ) and those of the left moving waves will have a negative real part (i.e., $\Re\left(K_{m n}\right)<0$ ). $P_{m n}(x)$ is referred to as a normal mode because it satisfies the impedance boundary condition along the right and left sidewall and can exist separately or in combination with other normal modes in the duct. Each normal mode can be shown to satisfy a reduced form of the convected Helmholtz equation

$$
P_{m n}^{\prime \prime}(x)+\left[\left(k-K_{m n} M\right)^{2}-K_{m n}^{2}-\left(\frac{m \pi}{H}\right)^{2}\right] P_{m n}(x)=0
$$

where a time dependency of the form $e^{i \omega t}$ has been assumed. Equation (2) is easily derived by substituting Eq. (1) into the linearized Euler equations for homentropic flow and eliminating the acoustic particle velocities to obtain a single ordinary differential equation on the normal acoustic pressure modes. Because the left sidewall is rigid, the boundary condition is

$$
P_{m n}^{\prime}(W)=0
$$

and the Myers local-reacting wall impedance boundary condition ${ }^{18}$ is applied along the surface of the liner

$$
P_{m n}^{\prime}(0)=\frac{i k}{\zeta}\left[1-\frac{M K_{m n}}{k}\right]^{2} P_{m n}(0)
$$

Equations (2)-(4) constitute a two-point boundary value problem (BVP) that can be solved in closed form to obtain the value of the normal acoustic pressure mode, $P_{m n}(x)$

$$
P_{m n}(x)=\cos \left(\lambda_{m n} x\right)+\tan \left(\lambda_{m n} W\right) \sin \left(\lambda_{m n} x\right), \quad \lambda_{m n}^{2}=\left(k-K_{m n} M\right)^{2}-\left(K_{m n}\right)^{2}-\left(\frac{m \pi}{H}\right)^{2}
$$

Substituting Eq. (5) into Eq. (4) and then solving for $\zeta$ gives

$$
\zeta=\frac{i k\left(1-\frac{M K_{m n}}{k}\right)^{2}}{\sqrt{\left(k-K_{m n} M\right)^{2}-\left(K_{m n}\right)^{2}-\left(\frac{m \pi}{H}\right)^{2}} \tan \left(W \sqrt{\left(k-K_{m n} M\right)^{2}-\left(K_{m n}\right)^{2}-\left(\frac{m \pi}{H}\right)^{2}}\right)}
$$

Equation (6) is used in conjunction with the measured quantities $\left(M, K_{m n}\right.$, and $\left.m\right)$ and duct geometry $(H, W)$ to determine the unknown liner impedance. As illustrated in Eq. (6), only a single axial propagation constant, $K_{m n}$, need be measured to obtained the unknown liner impedance. Here, we use Pronys method to extract a several axial propagation constants, $K_{m n}$. As shown later, Pronys method gives accurate predictions for the axial propagation constants of lower order (i.e., least attenuated) modes. The following section provides a brief description of the impedance eduction procedure. 


\section{Summary of Impedance Eduction Procedure}

The method for educing the impedance is now summarized:

1. From a measurement of the static temperature in the duct, compute the sound speed, $c_{0}$, and from a measurement of the total and static pressure in the duct, compute the mean flow Mach number, $M$.

2. Use the source drivers to drive a dominant vertical mode, $m, 20 \mathrm{~dB}$ (or more) above all other vertical modes. This value of $m$ is recorded and used as input to the reduced form of the convected Helmholtz equation [Eq. (2)].

3. Compute the freespace wavenumber, $k=\omega / c_{0}=2 \pi f / c_{0}$, for each excitation frequency, $f$.

4. Using the lid microphone data, extract a single axial wavenumber, $K_{m n}$, for the dominant vertical mode, $m$.

(a) If the measured slopes of the sound pressure level and phase decay rates are constant, then use the method presented in Ref. 4 to extract $K_{m n}$. This method for extracting the axial propagation constant is relatively straightforward but is only applicable when the sound pressure level and phase decay rates are constant.

(b) If these slopes are not constant, the sound field is contaminated with multiple modes and/or reflections. For this case, use the Prony method ${ }^{12-14}$ to extract a single axial wavenumber, $K_{m n}$, from the lid microphone data. The Prony method for extracting the axial propagation constant is less robust than the method presented in Ref. 4. Thus, the Prony method is only recommended in those cases for which the method used in Ref. 4 is not applicable.

5. Given the above inputs: $H, W, k, K_{m n}, m$, and $M$, perform the impedance eduction using Eq. (6).

\section{The Test Liner}

Two single-layer, perforate-over-honeycomb liner samples are used in this study, one for the GFIT and the other for the CDTR. These liner samples are representative of single-degree-of-freedom liners currently used in aircraft engine noise reduction. Figure 6 is a schematic of a typical liner sample. The GFIT liner sample is $51 \mathrm{~mm}$ wide and $610 \mathrm{~mm}$ long, whereas the CDTR sample is $381 \mathrm{~mm}$ wide and $813 \mathrm{~mm}$ long. Each liner sample consists of a $38.1 \mathrm{~mm}$ deep honeycomb core bonded on the backside to a $1 \mathrm{~mm}$ thick solid sheet and on the flow side to a $1 \mathrm{~mm}$ thick perforated plate. The perforate is 8.7 percent open area, and the holes in the plate are $1 \mathrm{~mm}$ diameter. The expectation is that the impedance spectra educed in the two flow ducts (GFIT and CDTR) should be nearly identical if the new, higher-order mode impedance eduction theory is valid.

\section{Results}

There are two primary goals for the current investigation. First, impedance spectra educed in the CDTR with the current higher-order mode theory are compared with those educed in the GFIT by using a benchmark impedance eduction method ${ }^{3}$ to demonstrate that the higher-order mode theory is valid. This comparison is initially performed when the $(0,0)$ mode is dominant in the upstream hard wall section of the CDTR, as is the case for all of the GFIT data. In addition, comparisons are made between these impedance spectra and those educed in the CDTR when two higher-order vertical modes (the $(1,0)$, and the $(2,0)$ mode) are dominant (sequentially, one at a time) in the upstream hard wall section. The impedance spectra are compared to determine whether the liner impedance is dependent on the vertical mode order of the incident wave. The benchmark validation impedances are educed using data acquired in the GFIT, where $H=63.5 \mathrm{~mm}, W=50.8 \mathrm{~mm}$, and a $406.4 \mathrm{~mm}$-long liner sample $\left(L_{2}-L_{1}=406.4 \mathrm{~mm}\right)$ is mounted in the upper wall. The higher-order mode theory is evaluated using data acquired in the CDTR. For the frequency range of interest $(0.3$ to $2.5 \mathrm{kHz})$, a number of higher-order horizontal modes [up to order $2(n=2)$ ] are cut on in the rigid wall sections of the CDTR, whereas only the plane wave mode exists in the rigid wall sections of the GFIT.

Figures 7-9 compare the impedance spectra educed in the CDTR for each targeted mode $(m=0, m=1$, and $m=2$ ) to that educed in the GFIT at Mach 0.0, 0.3, and 0.5, respectively. Educed impedances are plotted as a function of the excitation frequency, $f$, in $\mathrm{kHz}$. Each figure provides four curves. The first three are depicted with symbols that correspond to the educed values (either normalized resistance or normalized reactance) from the higher-order mode theory by using data acquired in the CDTR. The blue diamonds provide normalized impedances educed with a dominant vertical mode order of zero $(m=0)$. The red squares and green triangles provide the corresponding normalized impedances educed with dominant vertical mode orders of 1 and $2(m=1$ and $m=2)$, respectively. The 
last curve on each figure is a solid black line. This line represents the normalized impedance spectrum educed using data acquired in the GFIT. As indicated earlier, these GFIT data are used as the benchmark for comparison with the CDTR results.

All CDTR data presented in this report are acquired with a reference sound pressure level (SPL) of $130 \mathrm{~dB}$. This level was chosen because this is the level used in the GFIT test. For tests conducted in the CDTR, this reference level corresponds to the incident SPL of the selected dominant mode, i.e., the $(0,0),(1,0)$, or $(2,0)$ mode. For GFIT tests, this reference level corresponds to the total SPL measured at an axial plane $203 \mathrm{~mm}$ upstream of the liner leading edge. For the frequency range of interest, only the plane wave mode (the $(0,0)$ mode) propagates in the GFIT rigid wall sections upstream and downstream of the liner test section. GFIT data are acquired in $0.2 \mathrm{kHz}$ increments from 0.4 to $3.0 \mathrm{kHz}$ (a solid line is used solely to depict the fact that the GFIT approach is unique, and the corresponding normalized impedances are used as a benchmark), whereas CDTR data are acquired in $0.1 \mathrm{kHz}$ increments from 0.3 to $2.5 \mathrm{kHz}$. Note that CDTR data are only presented at frequencies where the selected source mode $[(0,0),(1,0)$, or $(2,0)]$ is cut on.

A number of interesting features are evident in Fig. 7-9. First, the normalized resistance is observed to increase with increasing Mach number, from approximately 0.2 at Mach 0.0 to 1.2 at Mach 0.5. This is expected for a conventional perforate-over-honeycomb liner. Also, the reactance is dominated by a $-\cot (k d)$ behavior. To discuss additional features of these graphs, we further divide the impedance spectra into three frequency ranges (a low, a mid or intermediate, and a high frequency range). In the low frequency range the excitation frequency is less than $0.6 \mathrm{kHz}$, in the $\mathrm{mid} /$ intermediate frequency range the excitation frequency is between $0.6 \mathrm{kHz}$ and $1.9 \mathrm{kHz}$, and in the high frequency range, the excitation frequency is $2.0 \mathrm{kHz}$ or greater.

First, consider the low frequency range. Significant separation occurs between the GFIT and CDTR results when there is flow (see Figs. 8 and 9). This separation between GFIT and CDTR results increases with the flow Mach number and is not observed in zero flow (Fig. 7). As the impedance spectra for the liners used in this study are expected to be smooth curves, the educed resistance spectra with flow are assumed to be incorrect at these lower frequencies. The reactance spectrum is expected to follow a $-\cot (k d)$ behavior; thus, the CDTR $m=0$ results appear to be more correct. One conjecture for the problems with impedance eduction at these lower frequencies is that the wavelength of the excitation frequency (approximately $.85 \mathrm{~m}$ at $0.4 \mathrm{kHz}$ ) is close to the length of the liner (approximately 0.81 $\mathrm{m})$. This hypothesis will be further evaluated in future tests. In addition, the attenuation is observed to be low at these lower frequencies (in both ducts), which adds considerable uncertainty to the impedance eduction process.

Next, consider the high frequency range of the spectra in Fig. 7-9. There is unacceptable scatter in the educed impedance spectra in this frequency range. The cause of this scatter can be better explained by a detailed investigation of the mode energy distribution in the vertical modes. Figure 10 shows the mode energy distribution achieved in the CDTR rigid wall sections upstream and downstream of the liner sample section at Mach $0.0(M=0.0)$. Observe that the target sound power level of $130 \mathrm{~dB}$ in the upstream section is achieved up to about $2.0 \mathrm{kHz}$ (the beginning of the high frequency region), and that the target mode (the $(0,0)$ mode for this case) is well above all other modes in this section of the duct. As the frequency is increased above $2.0 \mathrm{kHz}$, the capability of the control system to achieve optimum mode separation begins to break down, as there are not enough acoustic drivers in the system in comparison to the number of cut-on modes. In the downstream hard wall duct section, a good portion of the energy in the $(0,0)$ mode has been reduced. Some of it has been absorbed, while a portion has been scattered into another mode (the $(0,1)$ in this case). Since the $(0,1)$ mode has the same $m$ order (i.e., $m=0)$ as the targeted mode, this is not a problem for the current impedance eduction method. However, the growth of the modes with different $m$ orders than the targeted mode is problematic and occurs primarily in the high frequency region. Trends similar to results in Fig. 10 are observed at Mach 0.3 and 0.5 .

Figure 11 provides a graph of the separation (difference in sound power level) that was achieved between a targeted mode and the next mode with the largest sound power level and a different $m$ order. Results in the figure are acquired at Mach 0.0. Note that at $0.5 \mathrm{kHz}$, the separation between the targeted mode $(0,0)$ (i.e., the solid blue diamond) and the mode with the next largest sound power level (in this case the $(1,0)$ mode) is approximately $32 \mathrm{~dB}$. Recall that an underlying assumption of the current impedance eduction theory is that the targeted $m$ order must be dominant over the liner test section. Thus, if the mode separation is insufficient at either end of the duct, the primary assumption of the higher-order mode impedance eduction theory is not satisfied. The data of Fig. 11 suggest that the results achieved with the $(2,0)$ mode as the targeted source mode at $1.0 \mathrm{kHz}$ is of concern because the mode separation is low (approximately $10 \mathrm{~dB}$ and $5 \mathrm{~dB}$ on the upstream and downstream ends, respectively). It also indicates that the mode separation achieved with each of the targeted source modes $[(0,0),(1,0)$, and $(2,0)$ modes $]$ may be insufficient at frequencies above about $2.0 \mathrm{kHz}$. This is especially true as the Mach number is increased to 0.3 (Fig. 12) and 0.5 (Fig. 13). Thus, Fig. 10-13 confirm that the primary problem in the high frequency region of Fig. 7-9 is the loss of 
control authority over the vertical modes. That is, there are not a sufficient number of drivers to increase the sound power level in the incident vertical mode so that it can be considered dominant. This is a violation of the higher-order mode theory upon which this analysis is performed (see Section IV).

Finally, we consider the mid/intermediate frequency range of the impedance spectra depicted in Fig 7-9 where the incident vertical mode is dominant, and the primary assumptions of the theory are satisfied (except at $1.0 \mathrm{kHz}$ where the mode separation is of concern). Figure 7 provides impedance spectra educed from data acquired at Mach 0.0 (GFIT data were not available for frequencies below $0.5 \mathrm{kHz}$ ). The GFIT (solid black line) and CDTR $m=0$ (blue diamonds) normalized impedances are in excellent agreement. At Mach 0.3 (Fig. 8), the comparison between GFIT and CDTR $m=0$ normalized resistance spectra is also quite good in this mid frequency range. The normalized reactance spectra comparison is even better. At Mach 0.5 (Fig. 9), the comparison between GFIT and CDTR $m=0$ results are still quite good for both normalized resistance and reactance. Figures 7-9 also provide the impedance spectra that are educed from complex acoustic pressure data acquired in the CDTR with dominant vertical mode orders of 1 and 2 (i.e., $m=1$ and $m=2$ ). These impedance spectra are observed to compare very well to each other and with those for a vertical mode order of $0(m=0)$. The favorable comparison of GFIT and CDTR educed impedance in the mid frequency range for the lowest order vertical mode $(m=0)$ indicates the validity of the higher-order mode impedance eduction theory. The good comparison of educed impedance between the vertical modes $(m=0, m=1$, and $m=2)$ also provides evidence that the impedance of the liner in a grazing flow environment is independent of the vertical mode order of the incident sound wave.

The ability to educe impedance for liners installed in the CDTR in the mid frequency range (where the primary assumptions of the theory are generally valid) is significant. It eliminates the requirement that a companion liner be fabricated for and tested in the GFIT, to determine the liner impedance. Perhaps more importantly, it allows a direct comparison between results achieved with both of the Langley flow ducts (GFIT and CDTR), such that they can be used in a complementary manner. For example, some of the CDTR results in the low frequency appear to be more in line with expectations than the corresponding GFIT results. Since the "true" impedance is rarely known, the ability to compare answers from the two flow ducts can be used to build confidence in educed impedance in this range. Also, the agreement between the impedance educed in the CDTR (in the mid frequency range) for different $m$ orders is also significant. This is a very encouraging result and indicates (at least for the liner that was used in this test) that the acoustic impedance of the liner in grazing incidence is independent of the vertical mode order of the sound field that is present over the liner. This suggests that a comparison of impedance spectra educed with different vertical mode orders can be used to determine the "true" impedance, i.e., spurious results attained with one vertical mode may not be present with another vertical mode. It also provides confidence that aeroacoustic duct propagation codes can be used to predict sound attenuation due to acoustic liners, as the impedance specified at the liner surface is independent of which vertical mode is dominant over the liner. Future investigations will explore the expectation that the same will hold for horizontal modes (i.e., those that vary in the coordinate direction perpendicular to the liner surface).

\section{Concluding Remarks}

The single mode method of impedance eduction has been extended to a larger ducts where the assumption of uniform grazing flow is more reasonable (than in smaller ducts) and where multiple higher-order modes may be cut on simultaneously in one or both cross-sectional directions. The method requires that only one of the modes be dominant in the coordinate direction perpendicular to the two rigid walls and that acoustic pressure data be available only along a linear array of microphones adjacent to the liner sample that span the sample length. The two primary objectives of the study that were mentioned in the Introduction have been achieved. First, the method was validated by placing a liner sample in a duct with large cross-sectional dimensions (the CDTR) and a second liner sample (with the same liner parameters) in a duct with much smaller cross-sectional dimensions (the GFIT) and demonstrating that the same impedance spectra is educed for each sample under similar test conditions. The high-order mode theory developed in this paper was used in the large duct, whereas a previously developed benchmark method was used in the small duct. The second objective was to demonstrate (using measured data) that each higher order vertical mode sees the same local-reacting impedance at the liner surface in a grazing flow environment. This was demonstrated using the theory presented in this paper, but only in the large duct where several higher-order vertical modes were cut on that could be separated. To the authors knowledge, this is the first time that this has been demonstrated using measured

data. Clearly, additional tests with other liners with different impedance spectra are needed to further confirm these findings. The effects of the mean boundary layer need also be incorporated into these results, and these studies are currently underway. 


\section{Acknowledgment}

The NASA Fixed Wing Project of the Fundamental Aeronautics Program funded this research.

\section{References}

${ }^{1}$ Kraft, R. E., "Theory and Measurements of Acoustic Wave Propagation in Multi-Segmented Rectangular Ducts," P.h.D. Thesis, University of Cincinnati, June 1976.

${ }^{2}$ Watson, W. R., Jones, M. G., Tanner, S. E., and Parrott, T. L., "A Finite Element Propagation Model for Extracting Normal Incidence Impedance in Nonprogressive Acoustic Wave Fields," Journal of Computational Physics, Vol. 125, Issue 1, Apr. 1996, pp. $177-186$.

${ }^{3}$ Watson, W. R., Jones, M. G., and Parrott, T. L., "Validation of an Impedance Eduction Method in Flow,"AIAA Journal, Vol. 37, No. 7, July 1999, pp. 818-824.

${ }^{4}$ Jones, M. G., Watson, W. R., Tracy, M. B., and Parrott, T. L., "Comparison of Two Waveguide Methods for Educing Liner Impedance in Grazing Flow." AIAA Journal, Vol. 42, No. 2, Feb. 2004, pp. 232-240.

${ }^{5}$ Jones, M., Watson, W., and Parrott, T., "Benchmark Data for Evaluation of Aeroacoustic Propagation Codes with Grazing Flow." AIAA Paper 2005-2853, May 2005.

${ }^{6}$ Watson, W. R., Jones, M. G., and Parrott, T. L., "Comparison of a Convected Helmholtz and Euler Model for Impedance Eduction in Flow," AIAA paper 2006-2643, May 2006.

${ }^{7}$ Jones, M. G., Watson, W. R., and Nark, D. M., "Effects of Flow Profile on Educed Acoustic Liner Impedance," AIAA Paper 2010-3763, June 2010.

${ }^{8}$ Armstrong, D. L., Beckemeyer, R. J., and Olsen, F. R., "Impedance Measurements of Acoustic Duct Liners with Grazing Flow," Journal of the Acoustical Society of America, Vol. 55, No. S1, Apr. 1974, p. S59.

${ }^{9}$ Watson, W. R., "A Method for Determining Acoustic Liner Admittance in a Rectangular Duct with Grazing Flow from Experimental Data," NASA TP-2310, July 1984.

${ }^{10}$ Watson, W. R., "A New Method for Determining Acoustic-Liner Admittance in Ducts with Sheared Flow in Two Cross-Sectional Directions," NASA TP-2518, June 1985.

${ }^{11}$ Parrott, T. L., Watson, W. R., and Jones, M. G., "Experimental Validation of a Two-dimensional Shear Flow Model for Determining Acoustic Impedance,” NASA TP-2679, May 1987.

${ }^{12}$ Jing, X., Peng, S., and Sun, X., "A Straightforward Method for Wall Impedance Eduction in a Flow Duct," Journal of the Acoustical Society of America, Vol. 124, No. 1, July 2008, pp. 227-234.

${ }^{13}$ Renou, Y. and Auregan Y., "Failure of the Ingard-Myers Boundary Condition for a Lined Duct: An Experimental Investigation," Journal of the Acoustical Society of America, Vol. 130, No. 1, July 2011, pp. 52-60.

${ }^{14}$ Watson, W. R., and Jones, M. G., "A Comparative Study of Four Impedance Eduction Methodologies Using Several Test Liners." AIAA Paper 2013-2274, June 2013.

${ }^{15}$ Gerhold, C., Cabell, R., and Brown, M., "Development of an Experimental Rig for Investigation of Higher-Order Modes in Ducts," AIAA Paper 2006-2637, May 2006.

${ }^{16}$ Gerhold, C. H., Brown, M. C., Jones, M. G., Nark, D., and Howerton, B. M., "Configuration Effects On The Acoustic Performance Of A Duct Liner", AIAA Paper 2008-2977, May 2008

${ }^{17}$ Gerhold, C. H., Brown, M. C., Jones, M. G., and Howerton, B. H., "Report on Recent Upgrades to the Curved Duct Test Rig at NASA Langley Research Center," AIAA Paper 2011-2896, June 2011.

${ }^{18}$ Myers, M. K., “On The Acoustic Boundary Condition In The Presence Of Flow,” Journal Of Sound And Vibrations, Vol. 71 No. 3 , pp. 429-434, Aug. 1980.

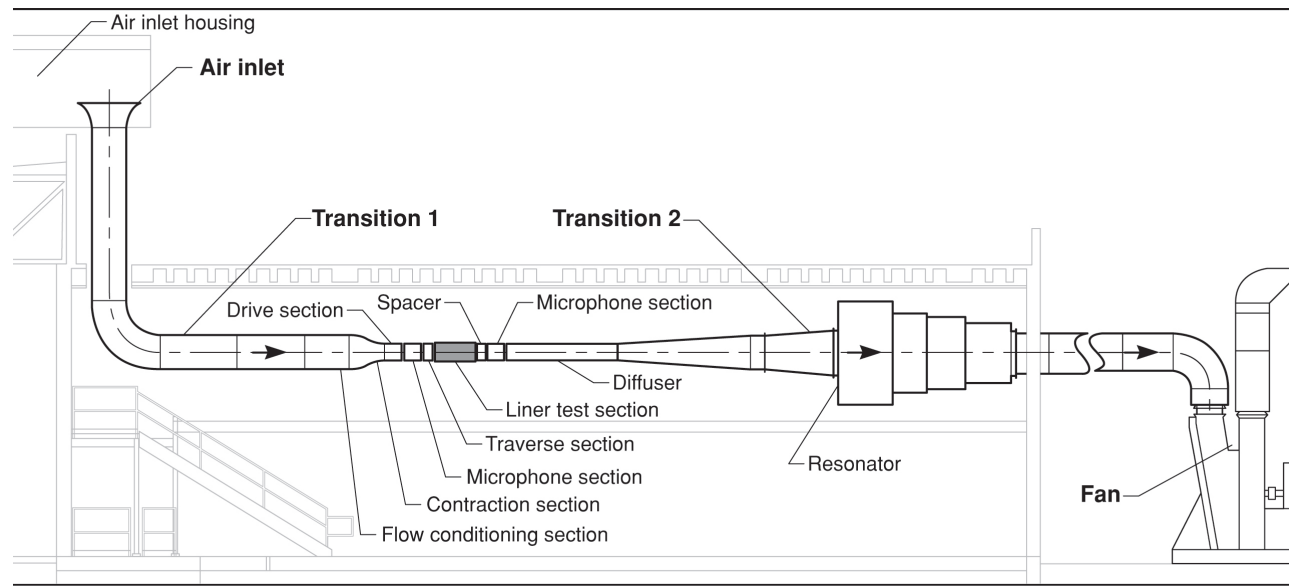

Figure 1. Curved Duct Test Rig layout in the Liner Technology Facility. 


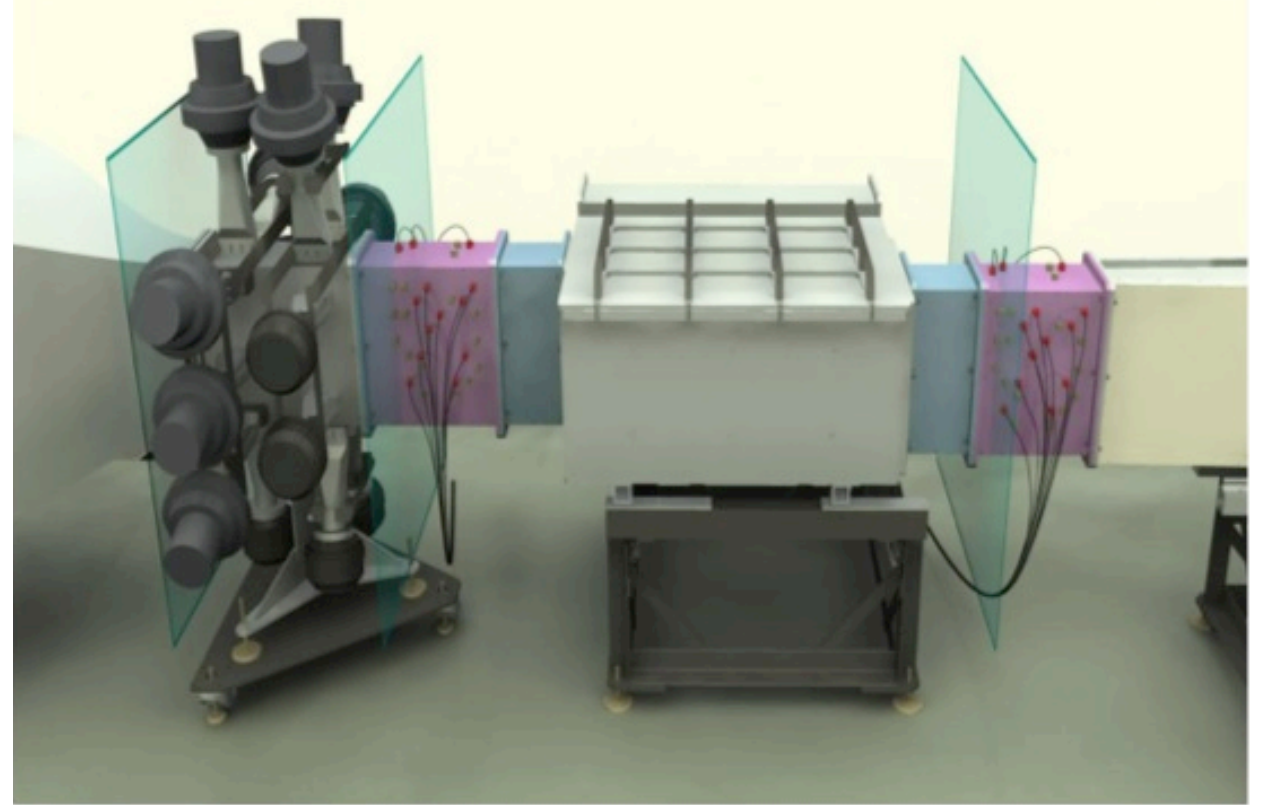

Figure 2. Curved Duct Test Rig test section showing sound source, upstream and downstream microphone arrays, and liner test section.

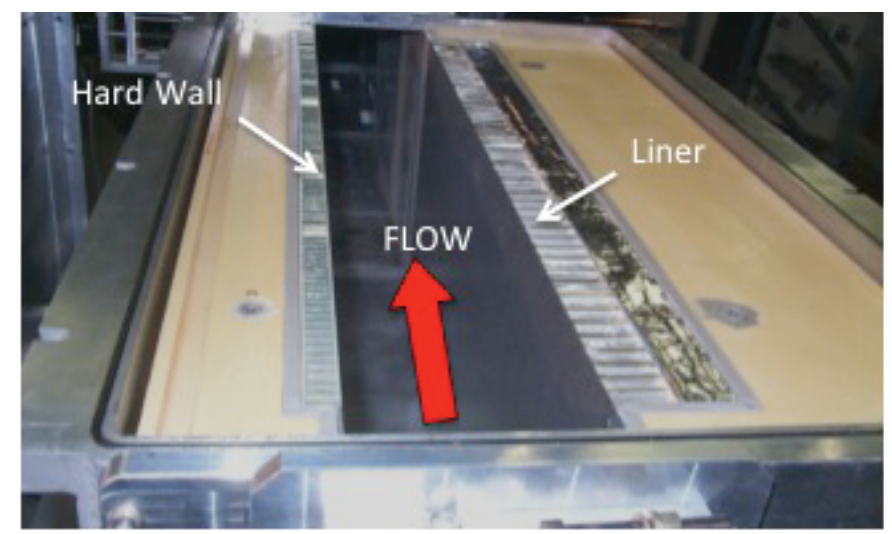

Figure 3. Curved Duct Test Rig liner test section with liner sample on right wall, hard wall opposite, top removed. 


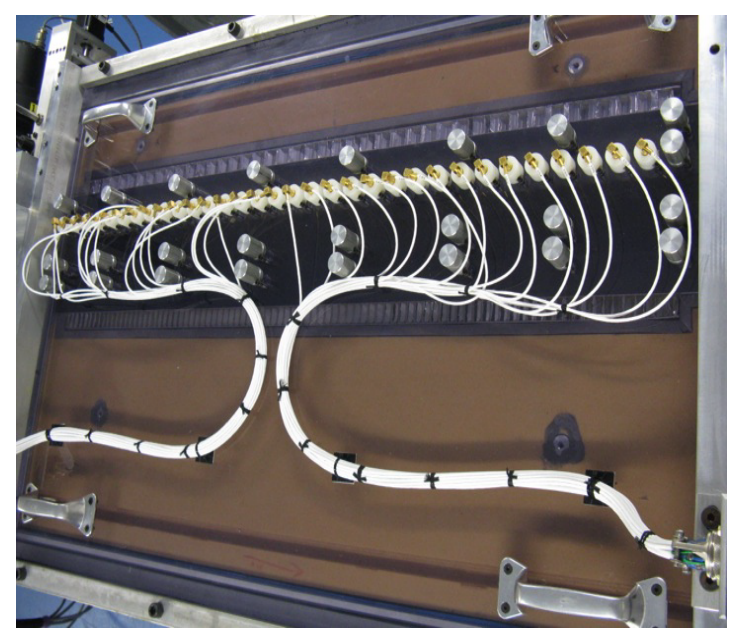

Figure 4. Photograph of Curved Duct Test Rig lid microphone array.

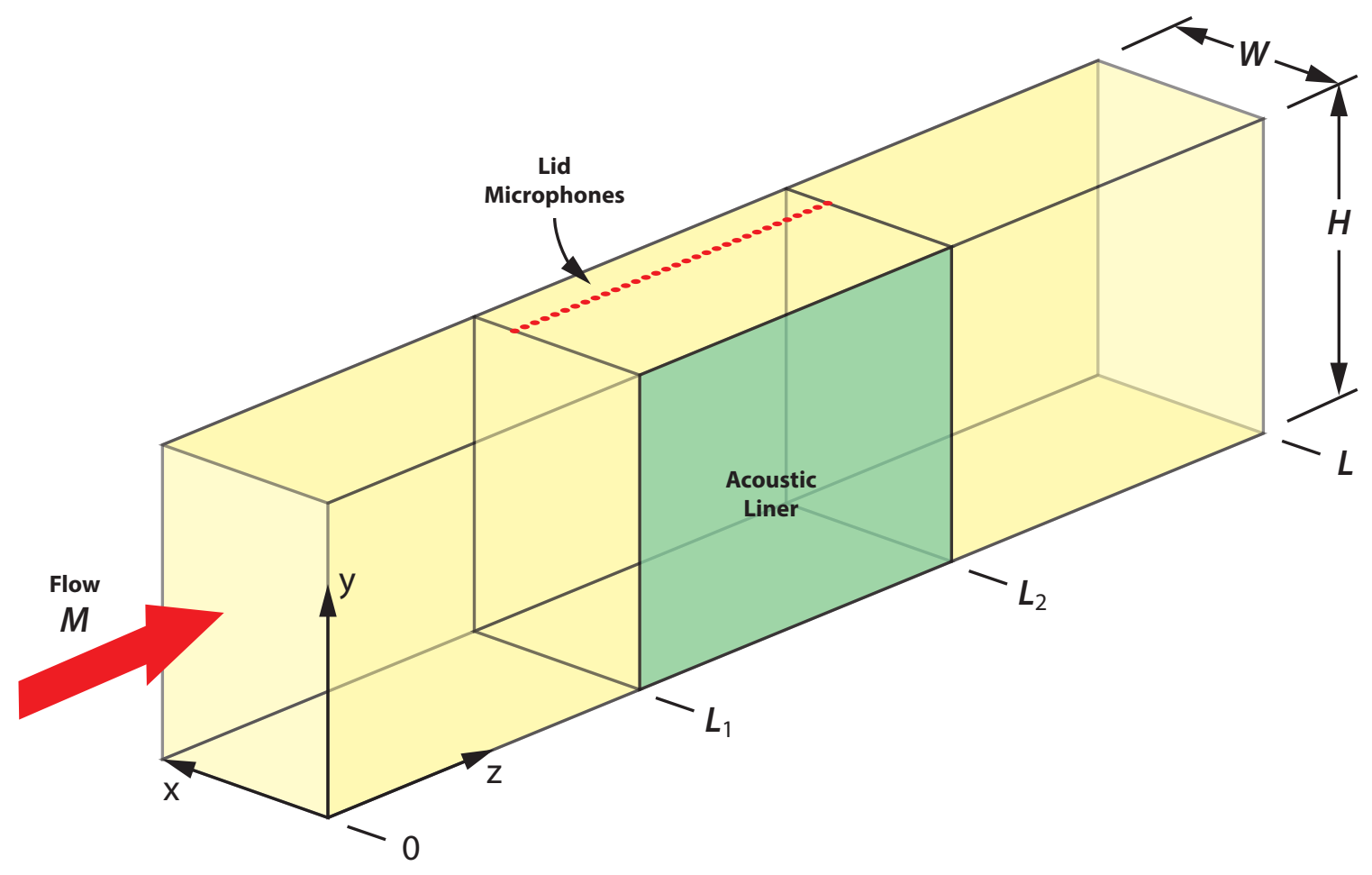

Figure 5. Schematic of 3D flow duct and Cartesian coordinate system. 


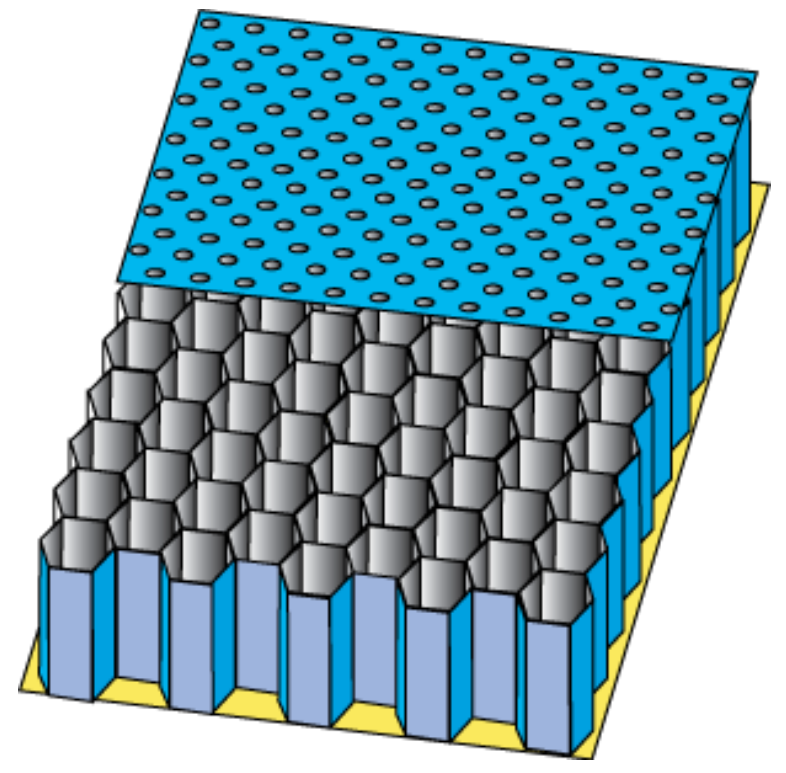

Figure 6. Schematic of the single-layer, perforate-over-honeycomb liner.

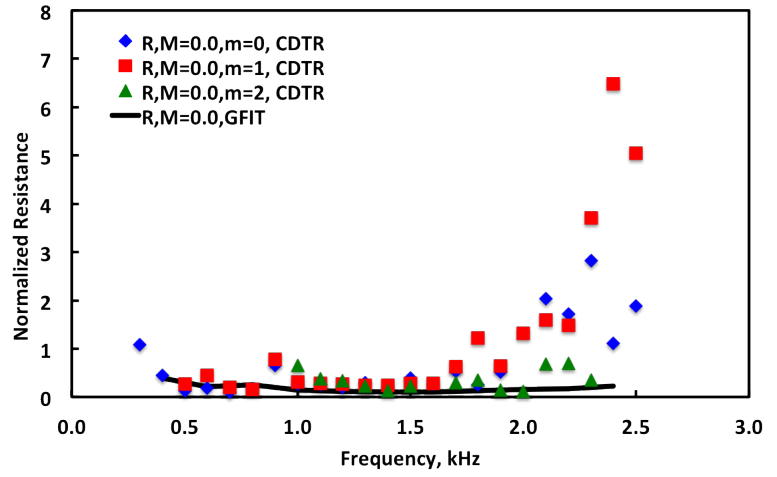

(a) Normalized resistance

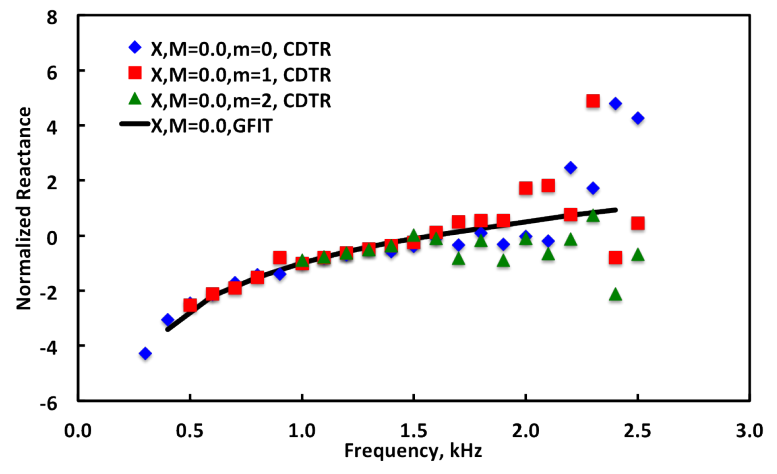

(b) Normalized reactance

Figure 7. Comparison of resistance spectra educed with the CDTR $(m=0,1$, and 2 dominant vertical modes) and GFIT for Mach 0.0.

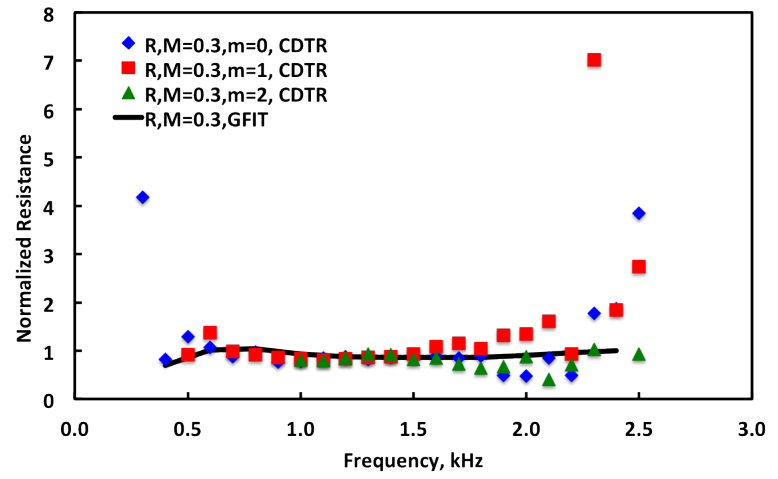

(a) Normalized resistance

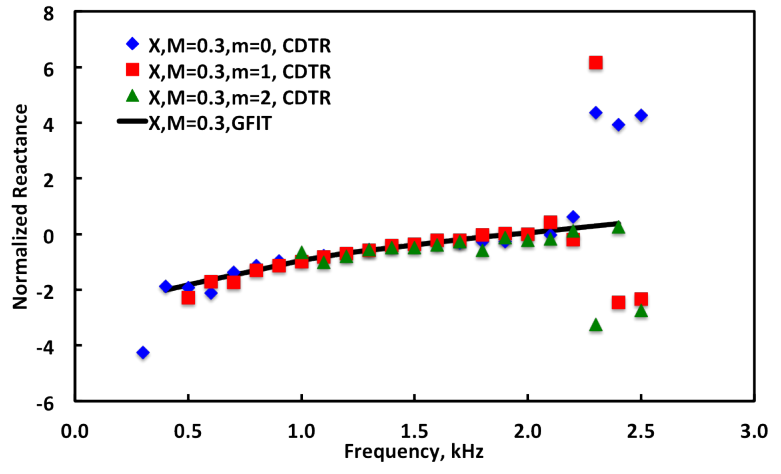

(b) Normalized reactance

Figure 8. Comparison resistance spectra educed with the CDTR $(m=0,1$, and 2 dominant vertical modes) and GFIT for Mach 0.3. 


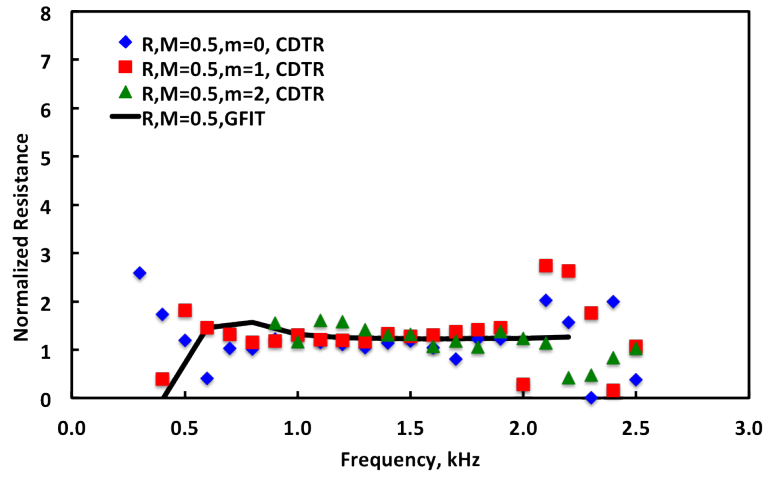

(a) Normalized resistance

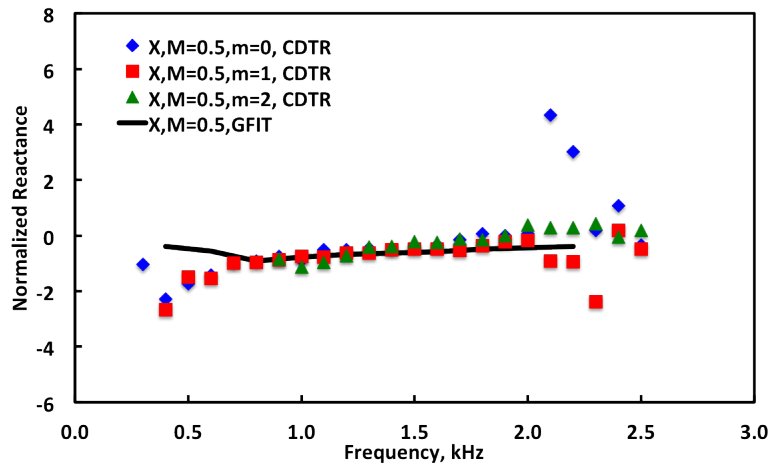

(b) Normalized reactance

Figure 9. Comparison resistance spectra educed with the CDTR $(m=0,1$, and 2 dominant vertical modes) and GFIT for Mach 0.5. 


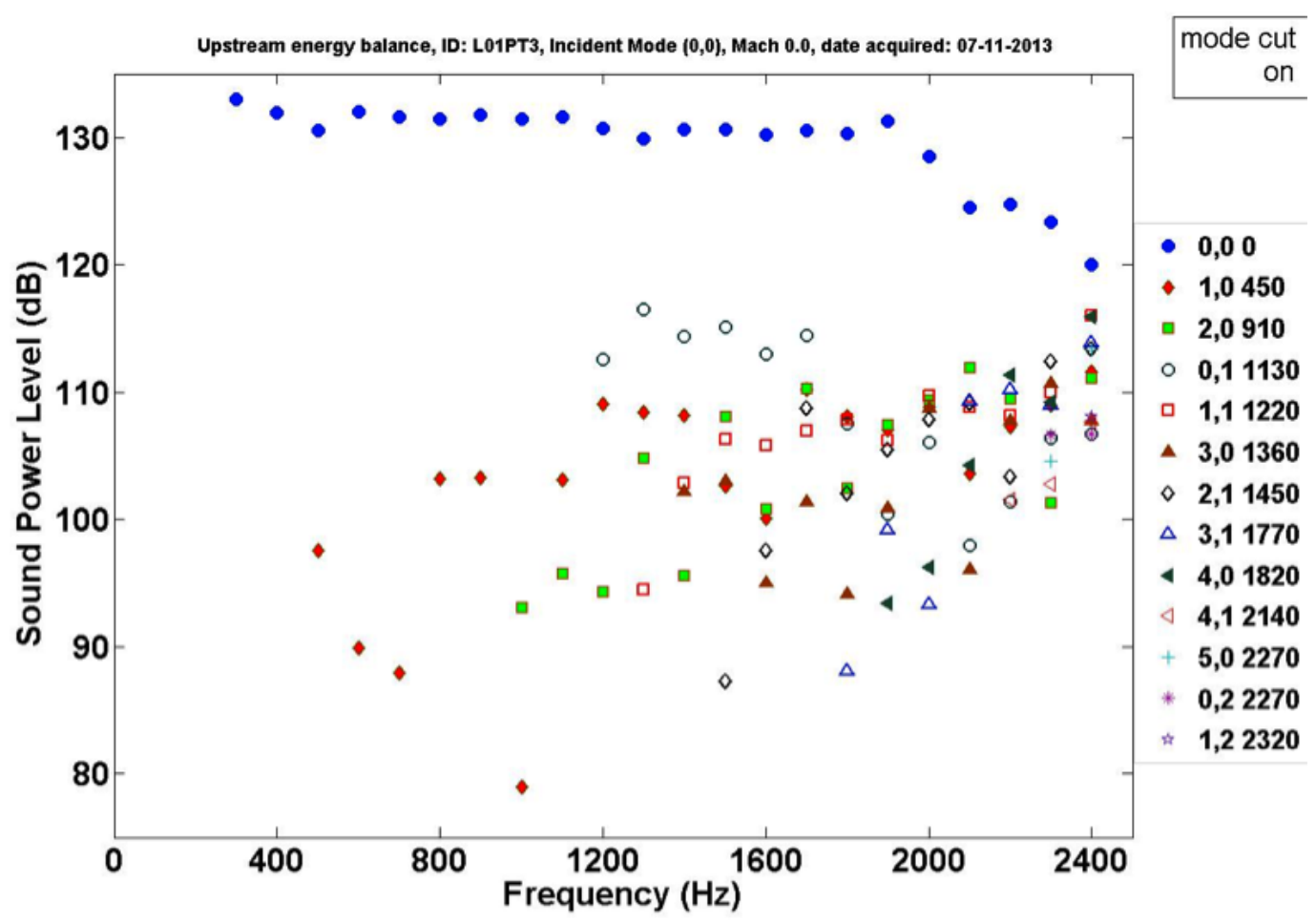

(a) Upstream of liner test section

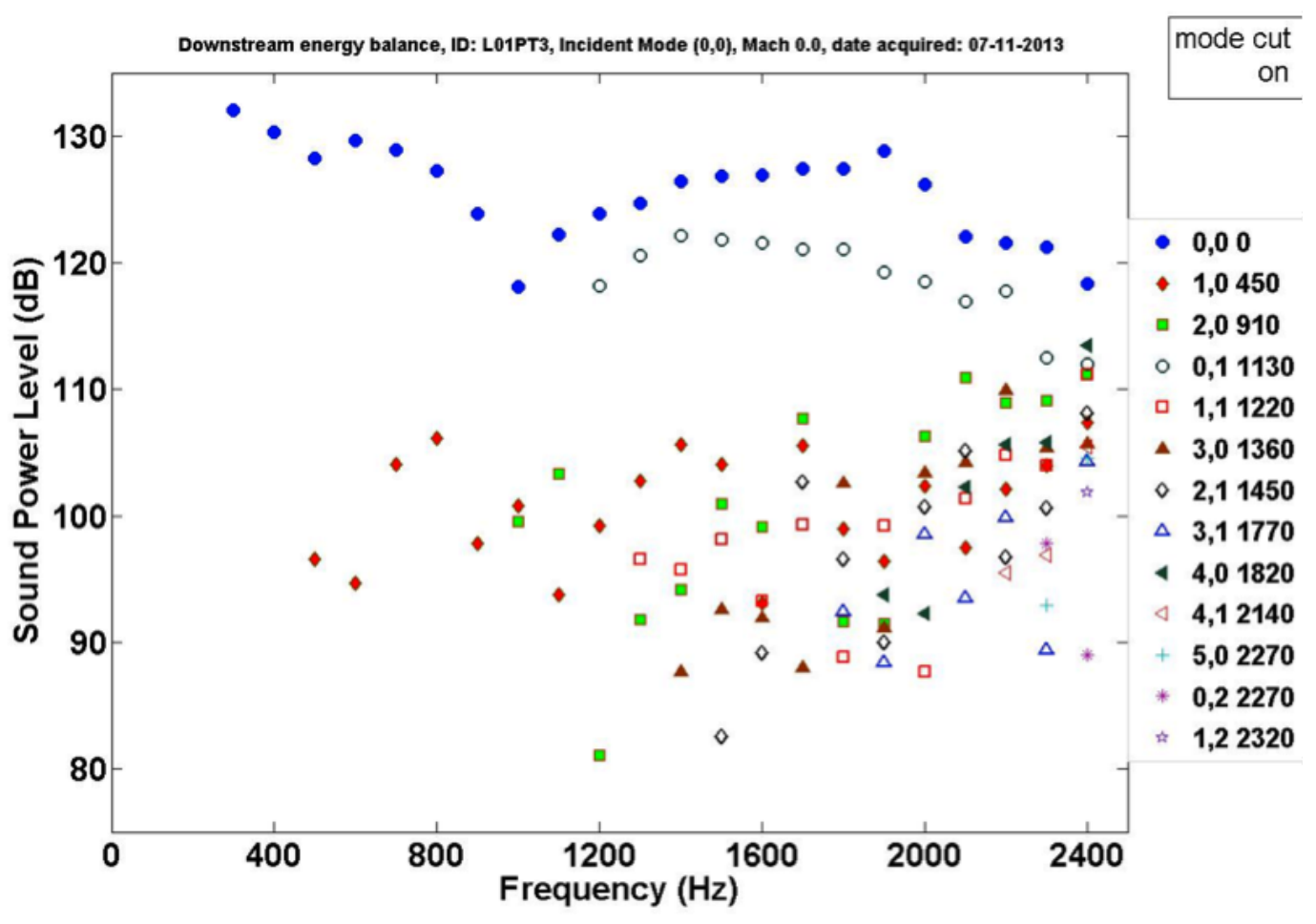

(b) Downstream of liner test section

Figure 10. Mode energy distribution in rigid wall sections of CDTR, Mach 0.0, mode $(0,0)$ incident. 


\section{L01 Liner, Mach 0.0}

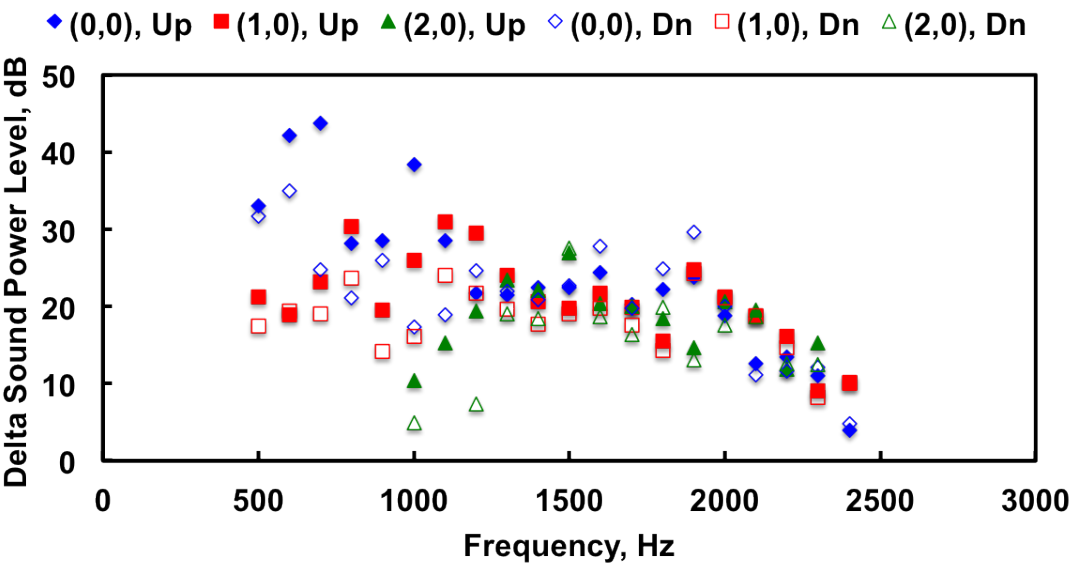

Figure 11. Difference between target mode $[(0,0),(1,0)$, or $(2,0)]$ and all modes with a different $m$ order. Solid symbols represent upstream mode separations, and open symbols represent downstream mode separations; Mach 0.0.

\section{L01 Liner, Mach 0.3}

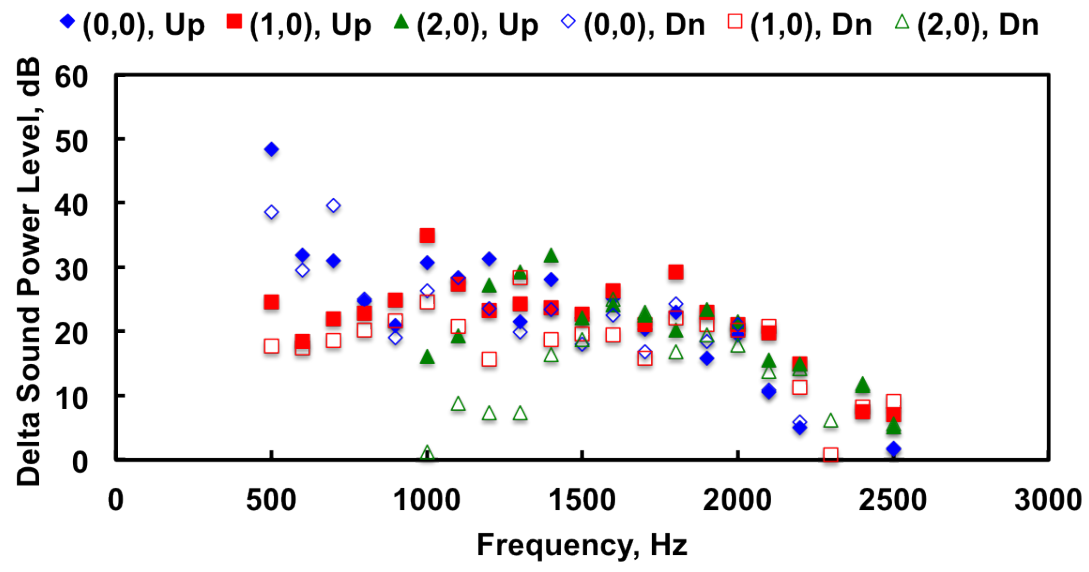

Figure 12. Difference between target mode $[(0,0),(1,0)$, or $(2,0)]$ and all modes with a different $m$ order. Solid symbols represent upstream mode separations, and open symbols represent downstream mode separations; Mach 0.3 . 


\section{L01 Liner, Mach 0.5}

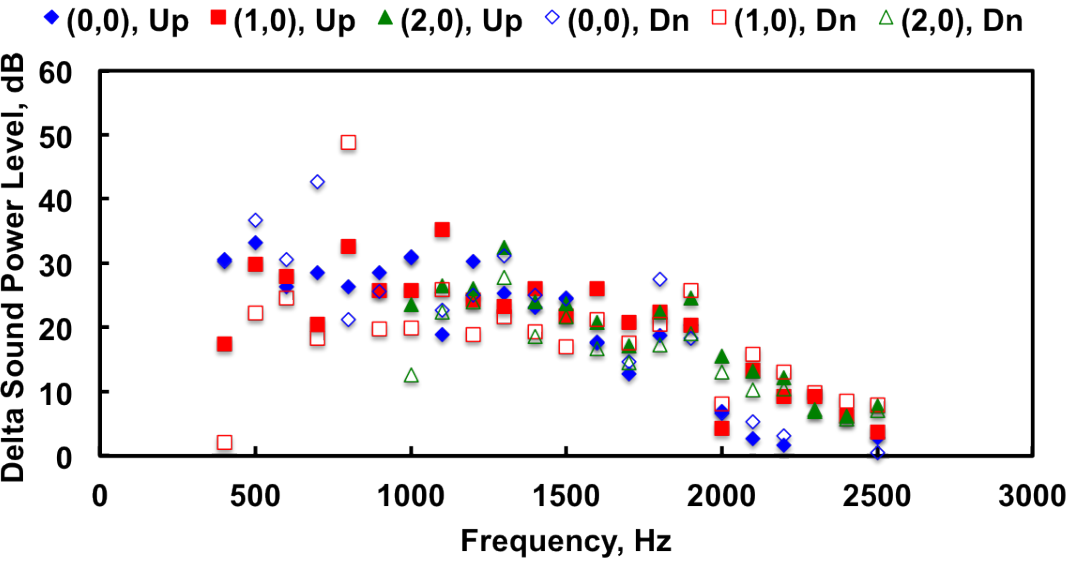

Figure 13. Difference between target mode $[(0,0),(1,0)$, or $(2,0)]$ and all modes with a different $m$ order. Solid symbols represent upstream mode separations, and open symbols represent downstream mode separations; Mach 0.5 . 